\title{
Correction to: Prediction Modeling Methodology
}

Frank J. W. M. Dankers, Alberto Traverso,

Leonard Wee, and Sander M. J. van Kuijk

Correction to: Chapter 8 in: P. Kubben et al. (eds.), Fundamentals of Clinical Data Science, https://doi.org/10.1007/978-3-319-99713-1_8

The original version of chapter 8 , was inadvertently published with error, the same has been updated as follows:

Incorrect:

Note that FNR is used in the next paragraph for the construction of the Receiver Operating Characteristic curve.

$\mathrm{FPR}=1-\mathrm{TPR}=1-$ sensitivity

$\mathrm{FNR}=1-\mathrm{TNR}=1-$ specificity

Correct:

Note that FPR is used in the next paragraph for the construction of the Receiver Operating Characteristic curve.

$\mathrm{FNR}=1-\mathrm{TPR}=1-$ sensitivity

$\mathrm{FPR}=1-\mathrm{TNR}=1-$ specificity

The updated online version of the original chapter can be found at https://doi.org/10.1007/978-3-319-99713-1_8 